\title{
Capital social y participación política de usuarios de Facebook
}

\section{Social capital and political participation of Facebook users}

\author{
Enrique Iván Noriega-Carrasco \\ Universidad Autónoma de Occidente, México \\ enrique.noriega@uadeo.mx \\ https://orcid.org/0000-0003-3002-337X \\ Evelia de Jesús Izábal de la Garza \\ Universidad Autónoma de Occidente, México \\ evelia.izabal@uadeo.mx \\ https://orcid.org/0000-0002-0268-5555
}

Recibido: 22/04/2021 Revisado: 05/06/2021 Aceptado: 25/06/2021 Publicado: 01/09/2021

\begin{abstract}
Resumen
México ha sido testigo de algunos movimientos sociales iniciados en las redes sociales, que fomentan conexiones que posibilitan el capital social, este último estrechamente relacionado con la participación política. Este poder ciudadano ligado a la democracia puede repercutir en las instituciones y el desarrollo económico. En este contexto, la investigación tiene como objetivo determinar la relación del capital social de los usuarios de Facebook con su participación política. Se realizó con un enfoque cuantitativo mediante una encuesta a 389 usuarios de Facebook del municipio de Culiacán, Sinaloa, por ser una de las ciudades mexicanas con un porcentaje mayor de usuarios de internet en el país con la finalidad de explicar el fenómeno sustentado en un análisis estadístico de correlaciones y regresiones lineales a partir de datos plasmados en escalas de Likert. Los hallazgos muestran que el capital social tradicional y en línea, en sus dimensiones bonding y bridging, a través de la participación política en línea de los usuarios de Facebook, influye significativamente en su participación política tradicional, esto es, se demostró que el capital social de usuarios de Facebook incide en la participación política fuera de línea. Se concluye que el capital social en redes sociales se constituye como una oportunidad para incrementar la participación política, en su forma tradicional o en línea, y con ello presionar a las autoridades a cumplir con las necesidades de la población.
\end{abstract}

\section{Palabras clave}

Capital social, medios sociales, participación política, internet, democracia, desarrollo participativo.

Forma sugerida de citar: Noriega-Carrasco, E. I., \& Izábal de la Garza, E. de J (2021). Capital social y participación política de usuarios de Facebook. Universitas-XXI, 35, pp. 129-150. https://doi.org/10.17163/uni.n35.2021.06 


\begin{abstract}
Mexico has witnessed some social movements initiated in social networks, which foster connections that enable social capital, the latter being closely related to political participation. This citizen power linked to democracy can have repercussions on institutions and economic development. In this context, this research has as its objective to determine the relationship of the social capital of Facebook users with their political participation. This investigation uses a quantitative approach through a survey applied to 389 Facebook users in the city of Culiacán, Sinaloa, as it is one of the Mexican cities with the highest percentage of internet users in Mexico in order to explain the phenomenon supported in a statistical analysis of correlations and linear regressions from data captured in Likert scales. The findings show that social capital in its online bonding dimension, as well as its online bridging and traditional dimensions through political participation online of Facebook users, significantly influence their traditional political participation, in other words, social capital of Facebook users affects political participation offline. It is concluded that social media social capital represents an opportunity to increase political participation, online and offline, and therefore pressure the authorities to meet the needs of their population.
\end{abstract}

\title{
Keywords
}

Social capital, social media, political participation, internet, democracy, participatory development.

\section{Introducción y estado de la cuestión}

Las redes sociales en línea han tomado relevancia científica desde principios del año 2000 (Kümpel et al., 2015) con movimientos que siguieron a la primavera árabe como la toma de Wall Street, los indignados y la revolución de las cacerolas (Knight, 2014), así como las campañas de Obama en 2008 y 2012, dando mayor interés en la vida cívica y política (Boulianne, 2015). México también ha sido testigo de algunos como el movimiento Zapatista, \#YoSoy132, la guardería ABC, los 43 estudiantes de Ayotzinapa, por mencionar algunos de los más relevantes (Cano, 2018).

Las redes sociales en línea son parte de la cotidianidad de este siglo XXI, su variedad permite el uso para contextos laborales, académicos, relaciones románticas, conectar intereses comunes (Ellison et al., 2007); Facebook ha tenido el mayor número de usuarios desde antes de 2015 seguido por YouTube, WhatsApp, Instagram, recientemente por TikTok, además de Snapchat y Twitter (ver Figura 1). 
Figura 1

Millones de usuarios a nivel mundial por año en redes sociales

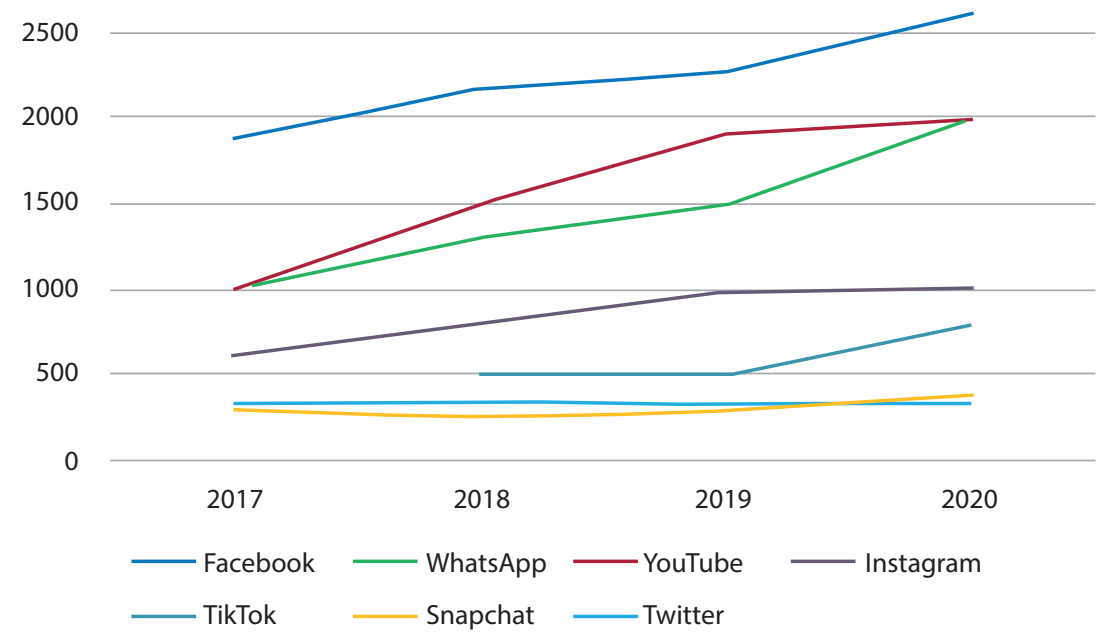

Fuente: Elaboración propia con datos obtenidos en cada red social (DataReportal, 2020; Facebook, 2020; Instagram, 2020; Snap Inc, 2020; YouTube, 2020).

Respecto a México, en 2018, el $99 \%$ de los usuarios de internet del país dijo usar estos sitios sociales, donde Facebook figuraba mayoritariamente con el 98 \% (Asociación de Internet.MX, 2018), una cantidad de ciudadanos considerable si se toma en consideración que el número de usuarios de internet en el país supera los 71 millones, equivalente a más del $60 \%$ de la población (INEGI, 2019); estos sitios están habilitados como nuevo medio de transmisión para información política así como otros sitios web y los medios tradicionales (Bode, 2016) con la distinción de que permite compartir información con conocidos y desconocidos pudiendo estar expuestos a contenido político de forma implícita posibilitando la generación de conocimiento sobre estos temas.

Como su nombre sugiere, estos sitios fomentan lazos sociales, ya sea manteniendo los existentes o generando nuevas conexiones que posibilitan el robustecimiento del capital social (Ellison et al., 2007) al permitir la existencia de comunidades digitales donde la presencia física no es necesaria (Gil de Zuñiga et al., 2012). El capital social es el reflejo de la relación 
recíproca entre compromiso cívico y confianza interpersonal que deriva en las instituciones y en participación (Brehm \& Rahn, 1997), se relaciona estrechamente con participación política (Skoric et al., 2009).

Ahora bien, la participación ciudadana es fundamental para la democracia (Arnstein, 1969; Valenzuela et al., 2016), en política busca influir en acciones de gobierno al afectar la toma de decisiones (Park, 2013). Partiendo de esta premisa, el presente documento contiene la información derivada de una investigación desarrollada en torno al comportamiento de los internautas que forman parte de la red social Facebook y como ello incide en acciones políticas dentro y fuera de línea.

El sustento de esta investigación radica en la relevancia de participación ciudadana y su vinculación con la democracia (Arnstein, 1969) en la actualidad. Con ella se busca el involucramiento de la sociedad para evitar ser pasivos, en aras de mantener el compromiso cívico y político (Irvin, 2004; King, 1998).

Los hallazgos de esta investigación ofrecen a las instituciones políticas y/o públicas, incluso fuera de México, los elementos para entender que la sociedad actual se encuentra en una transición en términos de e-participación ciudadana, lo que permitirá redefinir sus políticas y prácticas para fomentar esa colaboración bidireccional en aras del fortalecimiento de la democracia.

Finalmente, estudiar el caso en la ciudad de Culiacán en el estado de Sinaloa se justifica por ser una de las ciudades mexicanas con mayor porcentaje de usuarios de internet entre sus habitantes (INEGI, 2019) y por tener una participación política limitada, al ser uno de los municipios con menos participación en procesos electorales y estar en una entidad federativa que se encuentra por debajo de la media nacional en ese mismo indicador. A partir de lo anterior, esta investigación tiene como objetivo determinar la relación entre el capital social en los usuarios de redes sociales y su participación política; la investigación se delimitó a la red social Facebook, por ser la más utilizada en México (98\%), y por ser una de las dos redes sociales que se asocian con mayor frecuencia a movimientos sociales.

\section{Capital social y participación ciudadana en redes sociales}

El capital social hace referencia a las conexiones entre individuos; sus redes sociales, sus normas de reciprocidad y confianza (Putnam, 2001). A 
nivel individual es una inversión en relaciones sociales con retornos esperados, se realizan interacciones y forman redes para producir beneficios (Lin, 1999), generalmente referidos como recursos (Coleman, 1988). Quienes confían en esta inversión tienen mayor sentido de pertenencia a sus comunidades y toman un rol más activo (Shah et al., 2002). La definición de capital social de Bordieu y Wacquant (1992) agrega a estos recursos que sus características pueden ser físicas o virtuales al poseer una red duradera de relaciones de mutuo reconocimiento (Ellison et al, 2007).

Una reserva abundante de capital social supone la producción de una sociedad civil densa, la cual se ve como condición necesaria para la democracia liberal moderna (Fukuyama, 2001); produce consecuencias políticas promoviendo los procesos democráticos, la relación mencionada entre educación y participación política en el apartado interior puede ser causada por capital social (La Due Lake \& Huckfeldt, 1998). El capital social políticamente relevante se mide en términos de comunicación sobre política dentro de las redes recurrentes de interacción social del individuo (La Due Lake \& Huckfeldt, 1998).

Ya sea que se ejerza en su forma directa o representativa, la participación es parte fundamental de la democracia (Arnstein, 1969; Bakker \& de Vreese, 2011; Valenzuela et al., 2016). Sin la participación no son posibles las políticas y gobierno democrático (Darin, 2005), es el elixir de la vida para la democracia (van Deth, 2014).

La participación política sucede con el involucramiento en política y gobierno (Putnam, 2001), se refiere a toda acción que busca influir en las decisiones de los oficiales gobernantes o las políticas que crean e implementan, desde el voto, colaborar en campañas políticas, escribirle cartas a políticos o formar parte de una protesta (Verba et al., 2000), actividades de ciudadanos que afectan a la política (van Deth, 2014). Lo llevan a cabo personas en su rol de ciudadanos y no políticos o cabilderos profesionales, debe ser voluntaria y no obligada por ley, reglas o amenazas; involucra al gobierno, política o el Estado (Theocharis \& van Deth, 2018; van Deth, 2014).

Por otro lado, desde mediados de los años 90 del siglo pasado, el e-gobierno y la e-participación se han convertido en herramientas centrales de la administración pública y la interacción política (Kneuer \& Harnisch, 2016), contactar a gobernantes locales, estatales o federales, firmar una petición, escribir a un editor, comunicarse con un grupo político o comunitario, o bien, con sus miembros y hacer contribuciones políticas pueden ser reali- 
zados dentro y fuera de línea, incluso estas actividades se asocian entre sí, de forma que quienes participan en línea son muy propensos a hacerlo fuera de línea y viceversa (Schlozman et al., 2010). Las formas en que se puede participar en línea crean un modo de participación nuevo y distinto, que se ajusta a la taxonomía general de participación política (Theocharis \& van Deth, 2018).

Las redes sociales pueden proveer otras formas de exposición política a los no interesados sin tener que buscarla debido a que los interesados en política a menudo comparten información con sus redes en sitios como Facebook y Twitter. Si estas operan al igual que las redes sociales tradicionales se espera la estimulación para conversar e intercambiar información (Graber \& Dunaway, 2015). Es posible que la actividad expresiva en línea sea más influyente y pública que su contraparte fuera de línea, ya que publicar comentarios en un blog o en una página de redes sociales les proporciona una audiencia más amplia y es una experiencia más interactiva que usar un gafete o enviar una carta al editor de un periódico (Gibson \& Cantijoch, 2013).

Dar "me gusta" (o mecanismos equivalentes dentro de otras plataformas de redes sociales) y comentar contenido político en las redes sociales, requiere compromiso y movilización, puede ser un comportamiento de entrada para pensar o actuar sobre la política en otros ámbito, y requiere muy poco en términos de recursos al no tener costo monetario (Bode, 2017); al interactuar repetidamente se eleva la probabilidad de ver a contactos involucrándose en actividades políticas y a su vez seguir esa acción (Halpern et al, 2017).

En este contexto, el capital social permite el esparcimiento de información política y es esencial para los movimientos de carácter social y a su vez, estos generan capital social fomentando nuevas identidades y extendiendo redes sociales (Putnam, 2001). Un enfoque de capital social trata sobre sus características individuales con los poderes de sus conexiones sociales, amigos con ocupaciones de prestigio o conocidos con recursos instrumentales como proveer asistencia, influencia e información (Villalonga-Olives \& Kawachi, 2015). Otro enfoque lo aborda desde lo colectivo, es decir, los recursos incrustados en la estructura social que facilita las acciones de sus integrantes (Villalonga-Olives \& Kawachi, 2015).

Existen diversas formas de categorizar el capital social, una de ellas refiere la existencia de dos tipos de capital social: el horizontal que refleja los lazos que existen entre los grupos de individuos iguales o casi iguales; y el 
vertical (o linking) que se deriva de relaciones jerárquicas o desiguales debido a diferencias en el poder o base de recursos y el estatus (Islam et al., 2006). Esta investigación se aborda desde el capital social horizontal, el cual tiene dos formas básicas de acuerdo con Putnam: bonding y bridging; el primero describe los beneficios de las relaciones personales cercanas, que pueden incluir apoyo emocional, socorro físico u otros beneficios grandes (como la disposición a prestar una cantidad sustancial de dinero); mientras que el segundo describe los beneficios derivados de las relaciones y los contactos casuales, también puede conducir a resultados tangibles, como la información novedosa de conexiones distantes y visiones del mundo más amplias (Ellison et al., 2011). El bonding consiste en vínculos densos en un grupo, mientras el bridging se refiere a la fuerza de los lazos débiles dentro de una sociedad civil más amplia (Purdue, 2007).

Los vínculos sociales juegan un rol crucial en la información al público sobre política (Bode, 2016). La falta de redes sociales y vínculos con la comunidad hace que la participación sea indeseable y difícil (McLeod et al., 1999). Mientras que discutir de política con familiares y amigos es considerado un factor importante para la participación (Bakker \& de Vreese, 2011). El capital social políticamente relevante se genera en redes de relaciones sociales por su tamaño y por quienes discuten de política con frecuencia y experiencia (La Due Lake \& Huckfeldt, 1998). Sin embargo, no se puede esperar que en una sociedad cuya cultura cívica se basa en la desconfianza hacia la política y los políticos, una red social se convierta repentinamente en el escenario de debates democráticos (Meneses, 2015).

\section{Material y métodos}

Esta investigación busca medir la incidencia entre las variables: capital social en su modalidad tradicional (capital social fuera de línea u offline) y en línea (online) en sus dimensiones bridging y bonding, y participación política en su formato tradicional (participación política fuera de línea u offline) y en línea.

Específicamente se espera que la dimensión en línea bridging influya a la participación política en línea (H1) y tradicional (H2), así como también la dimensión en línea bonding (H3 y H4 respectivamente). Para el caso del capital social tradicional, la teoría suele vincularlo a la participación política 
tradicional, por lo que se espera una relación significativa entre ellos (H5) además de influir también a la participación política en línea (H6).

Para la obtención de correlaciones se requirieron las percepciones y motivaciones de la población en el municipio de Culiacán, Sinaloa, con la finalidad de explicar el fenómeno soportado en un análisis estadístico de correlaciones y regresiones lineales a partir de datos plasmados en escalas de Likert.

Se realizó la recolección de información a través de una encuesta integrada por variables de control, variables independientes relacionadas al capital social en sus modalidades tradicional y en línea en sus dimensiones bridging y bonding, y como variables dependientes la participación política en su formato tradicional y en línea.

Se diseñó un instrumento compuesto dividido en categorías equivalentes a las variables involucradas en el estudio. Una de las categoría mide el concepto de capital social en un índice utilizado por Gil de Zúñiga et al en 2012 (Gil de Zúñiga et al., 2012, 2017) basado en la escala de Williams (2006) quien ya contemplaba su aspecto dentro y fuera de línea bajo los términos bridging y bonding de Putnam (1995) y los lazos débiles y fuertes de Granovetter (1973), estas últimas dimensiones han sido adaptadas en otro índice para distintas localidades de aplicación, que van desde instituciones educativas hasta espacios virtuales (Ellison et al., 2007; Lee et al., 2014; Pang, 2018; Phua et al., 2017; Shane-Simpson et al., 2018; Vanden Abeele et al., 2018; You \& Hon, 2019) y de igual manera se retoma en esta investigación.

La siguiente categoría es la participación política dividida en tradicional y en línea. Es medida por la frecuencia de participación en actividades políticas en ambas modalidades y fueron retomadas de Gil de Zúñiga et. al. debido a su reincidencia en diversos artículos probando su validez (Gil de Zúñiga et al., 2012, 2014, 2017).

Para confirmar la validez del instrumento reflejada en la prueba piloto se realizó el análisis de fiabilidad con el total de la muestra. El instrumento en general mostró una $\alpha$ de Cronbach de 0.92. Al igual que en la prueba piloto se obtuvo la $\alpha$ por cada categoría, en donde las consistencias mayores fueron en capital social tradicional (0.89) y la participación política en línea (online) (0.89) (ver Tabla 1). 


\section{Tabla 1}

Análisis de fiabilidad por categoría del instrumento aplicado

\begin{tabular}{|l|c|}
\hline \multicolumn{1}{|c|}{ Categoría } & $\boldsymbol{\alpha}$ de Cronbach \\
\hline Capital social tradicional & 0.89 \\
\hline Capital social bridging en línea & 0.86 \\
\hline Capital social bonding en línea & 0.82 \\
\hline Participación política tradicional & 0.86 \\
\hline Participación política en línea & 0.89 \\
\hline
\end{tabular}

Fuente: Elaboración propia (2020).

Las tres medidas de capital social se analizaron factorialmente para asegurar que había distinción en sus dimensiones: capital social tradicional, capital social en línea bridging y capital social en línea bonding explicando el $65.6 \%$ de la varianza (ver Tabla 2).

Tabla 2

\section{Resultado de análisis factorial para ítems de capital social}

\begin{tabular}{|c|c|c|c|}
\hline & \multicolumn{3}{|c|}{ Carga factorial } \\
\hline & $\begin{array}{l}\text { CS } \\
\text { tradicional }\end{array}$ & $\begin{array}{l}\text { CS bridging } \\
\text { en línea }\end{array}$ & $\begin{array}{l}\text { CS bonding } \\
\text { en línea }\end{array}$ \\
\hline \multicolumn{4}{|l|}{ Capital social tradicional } \\
\hline $\begin{array}{l}\text { En mi comunidad, la gente se ayuda cuando hay un } \\
\text { problema }\end{array}$ & .827 & & \\
\hline En mi comunidad, las personas se cuidan mutuamente. & .861 & & \\
\hline En mi comunidad, hablamos sobre problemas comunales & .781 & & \\
\hline Creo que en mi comunidad se comparten valores & .838 & & \\
\hline Me siento en familia con las personas de mi comunidad & .787 & & \\
\hline \multicolumn{4}{|l|}{ Capital social bridging en línea } \\
\hline Me siento parte de la comunidad de Facebook & & .674 & \\
\hline Me interesa lo que pasa a mis amigos de Facebook & & .781 & \\
\hline Me siento cercano a mis amigos en Facebook & & .809 & \\
\hline $\begin{array}{l}\text { Interactuar en Facebook me motiva a probar cosas } \\
\text { nuevas }\end{array}$ & & .759 & \\
\hline
\end{tabular}




\begin{tabular}{|c|c|c|}
\hline $\begin{array}{l}\text { Interactuar en Facebook me hace sentir que formo parte } \\
\text { de una comunidad más grande }\end{array}$ & .723 & \\
\hline $\begin{array}{l}\text { En Facebook me pongo en contacto con gente nueva } \\
\text { frecuentemente }\end{array}$ & .509 & .437 \\
\hline \multicolumn{3}{|l|}{ Capital social bonding en línea } \\
\hline $\begin{array}{l}\text { Conozco personas en Facebook para que me ayuden a } \\
\text { resolver mis problemas }\end{array}$ & & .599 \\
\hline $\begin{array}{l}\text { Conozco personas en Facebook a quien pedir un } \\
\text { préstamo de emergencia }\end{array}$ & & .787 \\
\hline $\begin{array}{l}\text { Hay personas en Facebook con quien puedo hablar } \\
\text { cuando me siento solo }\end{array}$ & & .816 \\
\hline $\begin{array}{l}\text { Hay alguien en Facebook al que puedo acudir para } \\
\text { obtener consejos }\end{array}$ & .441 & .816 \\
\hline
\end{tabular}

Análisis factorial de los principales componentes con rotación varimax explicando el $65.6 \%$ de la varianza, los factoriales debajo de 0.40 no se muestran.

Fuente: elaboración propia (2020).

Este análisis factorial se realizó también a los ítems de participación política para asegurar distinción de dimensiones en sus categorías tradicional y en línea (online), explicando el $68.3 \%$ de la varianza (Tabla 3).

Tabla 3

\section{Resultado de análisis factorial para ítems de participación política}

\begin{tabular}{|l|c|c|}
\hline \multirow{2}{*}{\multicolumn{2}{|l|}{}} & \multicolumn{2}{c|}{ Carga factorial } \\
\cline { 2 - 4 } & $\begin{array}{c}\text { PP en } \\
\text { línea }\end{array}$ & $\begin{array}{c}\text { PP } \\
\text { tradicional }\end{array}$ \\
\hline Participación política en línea & & \\
\hline ¿Qué tan seguido utiliza el internet y las redes sociales para... & $\mathbf{. 6 6 4}$ & \\
\hline Firmar o compartir una petición en línea & $\mathbf{. 7 7 0}$ & \\
\hline Crear una petición en línea & $\mathbf{. 8 2 6}$ & \\
\hline Escribir a un político o funcionario de gobierno & $\mathbf{. 7 9 9}$ & \\
\hline Escribir a editores de periódicos & $\mathbf{. 6 8 2}$ & .432 \\
\hline Reclutarse como voluntario a causas políticas & $\mathbf{. 6 9 6}$ & .462 \\
\hline Iniciar un grupo político o página de redes sociales con causas políticas & \multicolumn{2}{|l}{} \\
\hline Participación política tradicional & & \\
\hline ¿Qué tan seguido... & .459 & \\
\hline Escribe cartas para grupos de noticieros & \multicolumn{2}{|l}{} \\
\hline
\end{tabular}




\begin{tabular}{|c|c|}
\hline Se comunica por carta, llamada o en persona con funcionarios & .607 \\
\hline $\begin{array}{l}\text { Publica anuncios políticos como prendedores... dona dinero a causas } \\
\text { políticas o campañas }\end{array}$ & .598 \\
\hline Asiste a audiencias o reuniones en el ayuntamiento & .788 \\
\hline Asiste a mítines, foros o debates políticos & .851 \\
\hline Asiste a protestas o marchas & 678 \\
\hline Participa en grupos políticos o campañas & .790 \\
\hline
\end{tabular}

Análisis factorial de los principales componentes con rotación varimax explicando el $68.3 \%$ de la varianza, los factoriales debajo de 0.40 no se muestran.

Fuente: Elaboración propia (2020).

La encuesta se aplicó totalmente en línea promoviéndose como publicidad de Facebook del 24 de septiembre al 25 de noviembre de 2019 en la ciudad de Culiacán a personas de 18 años en adelante, se obtuvieron 416 encuestas a personas sin distinción de sexo, nivel educativo, situación laboral ni estado civil. Del total se eliminaron 23 registros cuya edad indicaba ser menor a 18 años y cuatro más que resultaron duplicados restando un total de 389 .

Los datos para la obtención de resultados se procesaron con el software IBM SPSS Statistics versión 19 donde las variables se miden a partir de las categorías descritas anteriormente de forma que permita visualizar las correlaciones existentes y determinar si las aproximaciones teóricas son congruentes con los resultados.

\section{Análisis y resultados}

De los ítems de capital social, escalados a tres y por dimensiones, la dimensión con mayor puntaje resultó en su forma tradicional $(\mathrm{m}=2.14, \mathrm{~s}=$ $0.76)$, seguida por el bridging en línea $(\mathrm{m}=2.12, \mathrm{~s}=0.69)$ y la más baja fue su dimensión en línea bonding $(\mathrm{m}=1.84, \mathrm{~s}=0.88)$ (ver Tabla 4). Eso no implica que sean promedios altos solo que los primeros dos indican mayores incidencias de nivel medio-alto. 


\section{Tabla 4 \\ Estadístico de ítems en las categorías de capital social}

\begin{tabular}{|c|c|c|c|}
\hline & Media & Desv. Est. & Máximo \\
\hline Capital social tradicional & 2.14 & 0.76 & 3 \\
\hline En mi comunidad, la gente se ayuda cuando hay un problema & 2.97 & 0.97 & 4 \\
\hline En mi comunidad la gente se cuida mutuamente & 2.65 & 0.93 & 4 \\
\hline En mi comunidad, hablamos sobre problemas comunales & 2.40 & 0.99 & 4 \\
\hline Creo que en mi comunidad se comparten valores & 2.56 & 0.93 & 4 \\
\hline Me siento en familia con las personas de mi comunidad & 2.25 & 0.96 & 4 \\
\hline Capital social bridging en línea & 2.12 & 0.69 & 3 \\
\hline Me siento parte de la comunidad de Facebook & 2.27 & 0.92 & 4 \\
\hline Me interesa lo que pasa a mis amigos de Facebook & 2.60 & 0.92 & 4 \\
\hline Me siento cercano a mis amigos de Facebook & 2.40 & 0.93 & 4 \\
\hline Interactuar en Facebook me motiva a probar cosas nuevas & 2.17 & 0.95 & 4 \\
\hline $\begin{array}{l}\text { Interactuar en Facebook me hace sentir que formo parte de una } \\
\text { comunidad más grande }\end{array}$ & 2.12 & 0.98 & 4 \\
\hline En Facebook me contacto con gente nueva frecuentemente & 1.83 & 0.98 & 4 \\
\hline Capital social bonding en línea & 1.84 & 0.88 & 3 \\
\hline $\begin{array}{l}\text { Conozco personas en Facebook para que me ayuden a resolver } \\
\text { mis problemas }\end{array}$ & 1.69 & 0.94 & 4 \\
\hline $\begin{array}{l}\text { Conozco personas en Facebook a quien pedir un préstamo de } \\
\text { emergencia }\end{array}$ & 1.40 & 0.82 & 4 \\
\hline $\begin{array}{l}\text { Hay personas en Facebook con quien puedo hablar cuando me } \\
\text { siento solo }\end{array}$ & 1.80 & 0.99 & 4 \\
\hline $\begin{array}{l}\text { Hay alguien en Facebook al que puedo acudir para obtener } \\
\text { consejos }\end{array}$ & 1.70 & 0.99 & 4 \\
\hline
\end{tabular}

Fuente: Elaboración propia (2020).

Por su parte, la categoría de participación política tradicional muestra el nivel más bajo del estudio $(\mathrm{m}=1.33, \mathrm{~s}=0.64)$, y de forma muy contrastante la modalidad en línea tiene el más alto $(\mathrm{m}=2.27,2=0.45)($ ver Tabla 5$)$. 
Tabla 5

Estadístico de ítems en las categorías de participación política

\begin{tabular}{|c|c|c|c|}
\hline & Media & Desv. Est. & Máximo \\
\hline Participación política tradicional & 1.33 & 0.64 & 3 \\
\hline \multicolumn{4}{|l|}{ Que tan seguido ... } \\
\hline Escribe cartas para grupos de noticieros & 1.19 & 0.53 & 4 \\
\hline $\begin{array}{l}\text { Se comunica por carta, llamada o en persona con } \\
\text { funcionarios }\end{array}$ & 1.31 & 0.67 & 4 \\
\hline $\begin{array}{l}\text { Publica anuncios políticos como prendedores o } \\
\text { calcomanías, o bien, dona dinero a causas políticas o } \\
\text { campañas }\end{array}$ & 1.24 & 0.64 & 4 \\
\hline Asiste a audiencias o reuniones en el ayuntamiento & 1.28 & 0.64 & 4 \\
\hline Asiste a mítines, foros o debates políticos & 1.49 & 0.85 & 4 \\
\hline Asiste a protestas o marchas & 1.54 & 0.87 & 4 \\
\hline Participa en grupos políticos o campañas & 1.51 & 0.94 & 4 \\
\hline Participación política en línea & 2.27 & 0.45 & 3 \\
\hline \multicolumn{4}{|l|}{ Que tan seguido utiliza el internet y Facebook para ... } \\
\hline Firmar o compartir una petición en línea & 2.05 & 1.00 & 4 \\
\hline Crear una petición en línea & 1.59 & 0.87 & 4 \\
\hline Escribir a un político o funcionario de gobierno & 1.55 & 0.87 & 4 \\
\hline Escribir a editores de periódicos & 1.38 & 0.74 & 4 \\
\hline Reclutarse como voluntario a causas políticas & 1.38 & 0.80 & 4 \\
\hline $\begin{array}{l}\text { Iniciar un grupo político o página de redes sociales } \\
\text { con causas políticas }\end{array}$ & 1.35 & 0.77 & 4 \\
\hline
\end{tabular}

Fuente: Elaboración propia (2020).

Para el cumplimiento de las hipótesis propuestas fue necesario encontrar relaciones entre las variables de estudio, las cuales no pudieron identificarse simplemente con los estadísticos descriptivos y por tanto se calcularon las rhos de Spearman entre las categorías. 
Se sugirió una relación entre el capital social y la participación política, por tal motivo, se tomaron las categorías de tres dimensiones de capital social con las dos modalidades de participación política para observar sus correlaciones.

Al hablar de comunidades en línea y actividades en línea se anticipó obtener la mejor relación entre la participación política en línea y las dimensiones de capital social en línea, principalmente el bridging $(\varrho=0.27 ; \mathrm{p}<0.01)$ $(\mathrm{H} 1)$ seguida por el bonding $(\mathrm{Q}=0.26 ; \mathrm{p}<0.01)(\mathrm{H} 4)$. En tanto a la participación política tradicional solamente mostró una relación moderada con el capital social en línea bonding $(\mathrm{Q}=0.17 ; \mathrm{p}<0.01)(\mathrm{H} 3)$.

Es decir, el capital social en línea bridging no mostró ser significativo para la participación política tradicional (H2). Tampoco se encontró relación significativa entre el capital social y la participación política en sus formas tradicionales $(\mathrm{Q}=0.07 ; \mathrm{p}>0.05)(\mathrm{H} 5)$. El capital social tradicional se relacionó solamente con la participación política en línea de manera moderada $(\mathrm{Q}=0.15 ; \mathrm{p}<0.01)(\mathrm{H} 6)$.

Cabe mencionar que la relación entre los dos tipos de participación política (tradicional y en línea) es buena ( $\varrho=0.54 ; \mathrm{p}<0.01)$, permitiendo influir a la tradicional indirectamente a través de la en línea. Incluso mejor que las encontradas entre las dimensiones de capital social, donde la mayor fue entre las dimensiones en línea bridging y bonding ( $\mathrm{Q}=0.47 ; \mathrm{p}<0.01)$, seguido por el bridging y el tradicional $(\mathrm{Q}=0.32 ; \mathrm{p}<0.01)$, con el capital social tradicional y la dimensión bonding, aunque significativa, la relación fue más baja $(\mathrm{Q}=0.10 ; \mathrm{p}<0.05)$ (ver Figura 2).

Las regresiones lineales no solamente evidencian correlación entre variables, sino intentan predecir la influencia de una variable con otra. Por ello se realizaron los cálculos que complementaran la aprobación de las hipótesis mencionadas.

El capital social en línea bridging resultó la variable más influyente del estudio para la participación política en línea $(\beta=0.17$, p < 0.01) (H1), aunque no mostró ser significativa para la participación tradicional $(\beta=0.09, \mathrm{p}$ $>0.05)(\mathrm{H} 2)$. En el caso de la otra dimensión de capital social, el bonding, fue significativa tanto para la participación en línea $(\beta=0.13, \mathrm{p}<0.01)$ $(\mathrm{H} 3)$, como para la participación tradicional $(\beta=0.13, \mathrm{p}<0.01)(\mathrm{H} 4)$. 
Figura 2

Diagrama de correlaciones entre variables de capital social y participación política

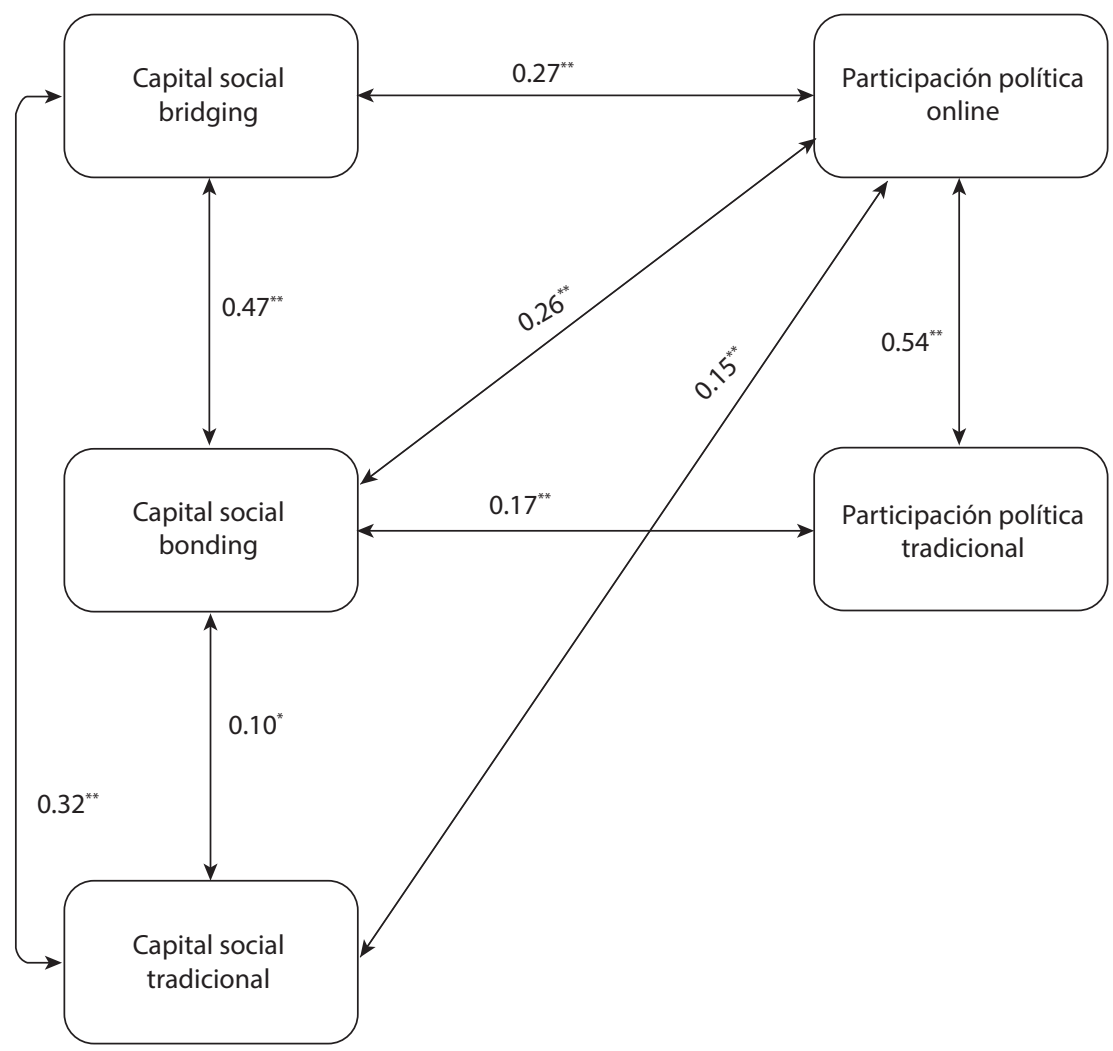

Los datos numéricos corresponden a coeficientes de correlación rho de Spearman (@) obtenidos por correlaciones bilaterales. ** $\mathrm{p} \leq 0.01 ; * \mathrm{p} \leq 0.05$

Fuente: Elaboración propia (2020)

En cuanto al capital social tradicional no resultó influyente para la participación política tradicional $(\beta=0.06, \mathrm{p}>0.05)(\mathrm{H} 5)$, pero si para la participación política en línea $(\beta=0.09, \mathrm{p}<0.01)(\mathrm{H} 6)$ (ver Tabla 6$)$. 


\section{Tabla 6}

\section{Predicción de participación política a partir del capital social}

\begin{tabular}{|l|c|c|}
\hline \multirow{2}{*}{\multicolumn{2}{|c|}{ Variables independientes }} & \multicolumn{2}{c|}{ Variables dependientes } \\
\cline { 2 - 3 } & \multicolumn{2}{|c|}{ Participación política } \\
\cline { 2 - 3 } & Tradicional & En línea \\
\hline Capital social tradicional & 0.06 & $0.09^{* *}$ \\
\hline$\Delta \mathrm{R}^{2}(\%)$ & 0.5 & 2.2 \\
\hline Capital social bridging en línea & 0.09 & $0.17^{* *}$ \\
\hline$\Delta \mathrm{R}^{2}(\%)$ & 0.9 & 7.1 \\
\hline Capital social bonding en línea & $0.13^{* *}$ & $0.13^{* *}$ \\
\hline$\Delta \mathrm{R}^{2}(\%)$ & 3.3 & 6.8 \\
\hline Total de $\mathrm{R}^{2}(\%)$ & 4.7 & 16.1 \\
\hline
\end{tabular}

Los datos contenidos en las celdas corresponden a los coeficientes Beta $(\beta)$ obtenidos por regresión de mínimos cuadrados ordinarios. ** $\mathrm{p} \leq 0.01 ; * \mathrm{p} \leq 0.05$.

Fuente: Elaboración propia (2020)

\section{Discusión y conclusiones}

Los resultados sugieren que a medida que los ciudadanos de Culiacán conozcan los asuntos públicos, ya sea a través de publicaciones compartidas o al participar en los llamados que surjan de Facebook como un ejemplo de red social, se tomarán las decisiones óptimas para su propio beneficio y, a pesar de no ser un mecanismo de participación formal, interactuar en las redes sociales puede presionar a las instituciones políticas a tomar decisiones que quizás de otra manera no realizarían. Cabe recordar que la participación política en redes sociales, aun sin tener forma física, cumple con ser un acto voluntario realizado por personas que no pertenecen al ámbito político, pero sí se ubican en él, lo que califica a dichas actividades como una forma válida de participación.

El análisis de capital social consideró tres dimensiones, el tradicional y en línea por bonding y bridging, dichas dimensiones mostraron relacionarse entre ellas mismas, esta correlación entre lo online y offline ya había sido encontrada en otras investigaciones (Gil de Zúñiga et al., 2017) aunque no consideraba las dimensiones bridging y bonding. 
Respecto a su relación con la participación política tradicional, y en contraste con lo que, establecido regularmente en las ciencias sociales, no se encontró relación significativa con el capital social tradicional (Gil de Zúñiga et al.2017). Se encontró una correlación fuerte entre ambas modalidades de participación política (Bakker \& de Vreese, 2011), quienes suelen o no participar en el formato tradicional también participan o no en línea, esta correlación fue la que presentó mayor fuerza.

Específicamente, se encontró que la variable que más influye a la participación política tradicional es su modalidad en línea. Esta participación política en línea es influida principalmente por el capital social bridging en línea, y este a su vez, por el capital social bonding en línea. Es decir, las relaciones en línea con personas cercanas, las de mayor confianza, influyen para participar políticamente, pero esta influencia se incrementa con la existencia de relaciones en línea casuales en el caso del uso de Facebook.

Estos hallazgos también se traducen a que la información publicada y compartida en las redes sociales como Facebook por familiares y amigos, pero sobre todo por las relaciones indirectas, influyen en la motivación a participar políticamente en línea, lo que hace propenso al individuo a repetir ese comportamiento de forma presencial, esto es, las formas de participación en línea están incidiendo en una forma de nueva de participación política (Theocharis \& van Deth, 2018).

La investigación cumple su objetivo al demostrar que el capital social, en sus dimensiones tradicional, bridging en línea y bonding en línea (al socializar la información, que puede contener asuntos públicos), incide en la participación política tradicional. Principalmente por las actividades que se realizan en línea.

El concepto tradicional de capital social no resultó predominante en las correlaciones como indican comúnmente las teorías, lo cual puede deberse al bajo nivel de participación presencial encontrada y que puede explicarse por las diferencias de esfuerzo respecto a las actividades en línea.

En la medida que se incremente la participación política, ya sea en su forma tradicional o en línea, se presiona a las autoridades a cumplir con las necesidades de la población, siendo las instituciones locales las más directas $\mathrm{y}$, a su vez, las indicadas para tomar las decisiones que diferencien quizás con lineamientos federales y permitan el desarrollo endógeno, o bien, que contribuyan a la creación de políticas públicas que se ajusten adecuadamente a la localidad. 
Este estudio logra dar pauta a la introducción de nuevas líneas de investigación que incluyan otras variables que busquen explicar, por ejemplo, si quienes no participan están desorientados por el fenómeno de las noticias falsas (fake news), o si factores como la percepción de corrupción o violencia provocan este efecto dado que Culiacán conserva un estigma relacionado al narcotráfico. Incluso no solamente en la participación, también su efecto en el capital social, así como también profundizar a través de métodos de etnografía virtual, también llamada etnografía digital.

Entre las limitaciones del estudio cabe destacar que los resultados están basados en usuarios de Facebook pues fue la plataforma utilizada para publicitarlo y que presentó mayor número de usuarios, sería pertinente conocer si en otras redes sociales y en otras entidades federativas más participativas se presentan distintas correlaciones. Los mecanismos de participación existentes y ejercidos en el lugar donde se aplique pueden utilizarse como apoyo a la información del estudio para fortalecer los datos y no concluir solamente con percepciones del ciudadano, las cuales, a pesar de no haber sido recolectadas en tiempos electorales, pueden verse influidas por alguna expectativa de mostrarse como buen ciudadano.

\section{Bibliografía}

Arnstein, S. R. (1969). A ladder of citizen participation. Journal of the American Planning Association, 35(4), 216-224. https://doi.org/10.1080/019 44366908977225

Asociación de Internet.MX. (2018). 14 Estudio sobre los hábitos de los usuarios de Internet en México 2018.

Bakker, T. P., \& de Vreese, C. H. (2011). Good news for the future? Young people, Internet use, and political participation. Communication Research, 38(4), 451-470. https://doi.org/10.1177/0093650210381738

Bode, L. (2016). Political News in the News Feed: Learning Politics from Social Media. Mass Communication and Society, 19(1), 24-48. https://doi.org/1 0.1080/15205436.2015.1045149

Bode, L. (2017). Gateway Political Behaviors: The Frequency and Consequences of Low-Cost Political Engagement on Social Media. Social Media + Society, 3(4), 205630511774334. https://doi.org/10.1177/2056305117743349 
Boulianne, S. (2015). Social media use and participation: a meta-analysis of current research. Information, Communication \& Society, 18(5), 524-538. https://doi.org/10.1080/1369118X.2015.1008542

Brehm, J., \& Rahn, W. (1997). Individual-Level Evidence for the Causes and Consequences of Social Capital. American Journal of Political Science, 41(3), 999. https://doi.org/10.2307/2111684

Cano, C. A. R. (2018). Communication in Movement and Techno-Political Media Networks: the case of Mexico. In Networks, Movements and Technopolitics in Latin America (pp. 147-175). Springer International Publishing. https://doi.org/10.1007/978-3-319-65560-4_8

Coleman, J. S. (1988). Social Capital in the Creation of Human Capital. American Journal of Sociology, 94(2), S95-S120. https://doi.org/10.1086/228943

Darin, D. (2005). Communication technology. S. Wight (ed.); First ed. UBC Press. DataReportal. (2020). DataReportal. https://datareportal.com/

Ellison, N. B., Steinfield, C., \& Lampe, C. (2007). The benefits of facebook "friends:" Social capital and college students' use of online social network sites. Journal of Computer-Mediated Communication, 12(4), 11431168. https://doi.org/10.1111/j.1083-6101.2007.00367.x

Ellison,N.B.,Steinfield,C.,\& Lampe,C.(2011).Connection strategies: Social capital implications of Facebook-enabled communication practices. New Media \& Society, 13(6), 873-892. https://doi.org/10.1177/1461444810385389

Facebook. (2020). Facebook Newsroom. https://about.fb.com/news

Fukuyama, F. (2001). Social capital, civil society and development. Third World Quarterly, 22(1), 7-20. https://doi.org/10.1080/01436590020022547

Gibson, R., \& Cantijoch, M. (2013). Conceptualizing and Measuring Participation in the Age of the Internet: Is Online Political Engagement Really Different to Offline? The Journal of Politics, 75(3), 701-716. https://doi. org/10.1017/S0022381613000431

Gil de Zúñiga, H., Barnidge, M., \& Scherman, A. (2017). Social media social capital, offline social capital, and citizenship: exploring asymmetrical social capital effects. Political Communication, 34(1), 44-68. https://doi.org/10 $.1080 / 10584609.2016 .1227000$

Gil de Zúñiga, H., Jung, N., \& Valenzuela, S. (2012). Social media use for news and individuals' social capital, civic engagement and political participation. Journal of Computer-Mediated Communication, 17(3), 319-336. https:// doi.org/10.1111/j.1083-6101.2012.01574.X 
Gil de Zúñiga, H., Molyneux, L., \& Zheng, P. (2014). Social media, political expression, and political participation: Panel analysis of lagged and concurrent relationships. Journal of Communication, 64(4), 612-634. https:// doi.org/10.1111/jcom.12103

Graber, D. A., \& Dunaway, J. (2015). Mass media and american politics (Ninth edit). SAGE Publications.

Halpern, D., Valenzuela, S., \& Katz, J. E. (2017). We face, i tweet: how different social media influence political participation through collective and internal efficacy. Journal of Computer-Mediated Communication, 22(6), 320-336. https://doi.org/10.1111/jcc4.12198

INEGI (2019). Encuesta Nacional sobre Disponibilidad y Uso de Tecnologías de la Información en los Hogares 2018. https://www.inegi.org.mx/programas/ dutih/2018/default.html

Instagram (2020). Instagram Business.

Islam, M. K., Merlo, J., Kawachi, I., Lindström, M., \& Gerdtham, U. G. (2006). Social capital and health: Does egalitarianism matter? A literature review. International Journal for Equity in Health, 5. https://doi. org/10.1186/1475-9276-5-3

Kneuer, M., \& Harnisch, S. (2016). Diffusion of e-government and e-participation in democracies and autocracies. Global Policy, 7(4), 548-556. https:// doi.org/10.1111/1758-5899.12372

Knight, P. T. (2014). The Internet in Brazil (First ed). AuthorHouse.

Kümpel, A. S., Karnowski, V., \& Keyling, T. (2015). News sharing in social media: a review of current research on news sharing users, content, and networks. Social Media + Society, 1(2), 205630511561014. https://doi. org/10.1177/2056305115610141

La Due Lake, R., \& Huckfeldt, R. (1998). Social capital, social networks, and political participation. Political Psychology, 19(3), 567-584. https://doi. org/10.1111/0162-895X.00118

Lee, E., Kim, Y. J., \& Ahn, J. (2014). How do people use Facebook features to manage social capital? Computers in Human Behavior, 36, 440-445. https:// doi.org/10.1016/j.chb.2014.04.007

Lin, N. (1999). Building a network theory of social capital. Connections, 22(1), 2851. https://bit.ly/3vFTOjo

McLeod, J. M., Scheufele, D. A., \& Moy, P. (1999). Community, communication, and participation: the role of mass media and interpersonal discussion in 
local political participation. Political Communication, 16(3), 315-336. https://doi.org/10.1080/105846099198659

Meneses, M. E. (2015). Ciberutopías. Democracia, redes sociales, movimientos red. Editorial Porrúa Primera ed.

Pang, H. (2018). Exploring the beneficial effects of social networking site use on Chinese students' perceptions of social capital and psychological wellbeing in Germany. International Journal of Intercultural Relations, 67(July), 1-11. https://doi.org/10.1016/j.ijintrel.2018.08.002

Park, C. S. (2013). Does Twitter motivate involvement in politics? Tweeting, opinion leadership, and political engagement. Computers in Human Behavior, 29(4), 1641-1648. https://doi.org/10.1016/j.chb.2013.01.044

Phua, J., Jin, S. V., \& Kim, J. (Jay). (2017). Uses and gratifications of social networking sites for bridging and bonding social capital: A comparison of Facebook, Twitter, Instagram, and Snapchat. Computers in Human Behavior, 72, 115-122. https://doi.org/10.1016/j.chb.2017.02.041

Purdue, D. (2007). Civil society, governance, social movements and social capital. In D. Purdue (Ed.), Civil Societies and Social Movements (1st ed., pp. 220-227). Routledge.

Putnam, R. D. (2001). Bowling alone: The collapse and revival of American community (1st Ed.). Simon \& Schuster Paperbacks.

Schlozman, K. L., Verba, S., \& Brady, H. E. (2010). Weapon of the strong? Participatory inequality and the internet. Perspectives on Politics, 8(2), 487509. https://doi.org/10.1017/S1537592710001210

Shah, D. V., Schmierbach, M., Hawkins, J., Espino, R., \& Donavan, J. (2002). Nonrecursive models of internet use and community engagement: questioning whether time spent online erodes social capital. Journalism \& Mass Communication Quarterly, 79(4), 964-987. https://doi. org/10.1177/107769900207900412

Shane-Simpson, C., Manago, A., Gaggi, N., \& Gillespie-Lynch, K. (2018). Between privacy and self-expression, and implications for social capital. Сотрuter in Human Behavior, 86, 276-288. https://cloudfront.escholarship.org/ $\mathrm{dist} / \mathrm{prd} /$ content/qt9sq7z6gz/qt9sq7z6gz.pdf

Skoric, M. M., Ying, D., \& Ng, Y. (2009). Bowling online, not alone: Online social capital and political participation in Singapore. Journal of Computer-Mediated Communication, 14(2), 414-433. https://doi.org/10.1111/j.10836101.2009.01447.x 
Snap Inc. (2020). Snap Investor Relations. https://investor.snap.com/overview/default.aspx

Theocharis, Y., \& van Deth, J. W. (2018). The continuous expansion of citizen participation: a new taxonomy. European Political Science Review, 10(01), 139-163. https://doi.org/10.1017/S1755773916000230

Valenzuela, S., Somma, N. M., Scherman, A., \& Arriagada, A. (2016). Social media in Latin America: deepening or bridging gaps in protest participation? Online Information Review, 40(5), 695-711. https://doi.org/10.1108/ OIR-11-2015-0347

van Deth, J. W. (2014). A conceptual map of political participation. Acta Politica, 49(3), 349-367. https://doi.org/10.1057/ap.2014.6

Vanden Abeele, M. M. P., Antheunis, M. L., Pollmann, M. M. H., Schouten, A. P., Liebrecht, C. C., van der Wijst, P. J., van Amelsvoort, M. A. A., Bartels, J., Krahmer, E. J., \& Maes, F. A. (2018). Does Facebook Use Predict College Students' Social Capital? A Replication of Ellison, Steinfield, and Lampe's (2007) Study Using the Original and More Recent Measures of Facebook Use and Social Capital. Communication Studies, 69(3), 272282. https://doi.org/10.1080/10510974.2018.1464937

Verba, S., Schlozman, K. L., \& Brady, H. E. (2000). Rational action and political activity. Journal of Theoretical Politics, 12(3), 243-268. https://doi. org/10.1177/0951692800012003001

Villalonga-Olives, E., \& Kawachi, I. (2015). The measurement of social capital. Gaceta Sanitaria, 29(1), 62-64. https://doi.org/10.1016/j.gaceta.2014.09.006

You, L., \& Hon, L. (2019). How social ties contribute to collective actions on social media: A social capital approach. Public Relations Review, May, 101771. https://doi.org/10.1016/j.pubrev.2019.04.005

YouTube (2020). YouTube Official for Press. https://blog.youtube/press 